\title{
Tratamiento en línea para usuarios de sustancias adictivas
}

\author{
Ricardo Sánchez Huesca', Liliana Berenice Templos Núñez' \\ I Centros de Integración Juvenil
}

La pandemia por COVID-19 ha modificado prácticas y conductas en amplios sectores de servicios, incluyendo la medicina, la psicología y las ciencias sociales. Las medidas sanitarias, indispensables para controlar contagios, hicieron que se suspendieran o disminuyeran los tratamientos cara a cara con usuarios de sustancias. Ante tal circunstancia surgió la necesidad de encontrar alternativas para continuar proporcionando atención, sobre todo al tener evidencia de que tener un Trastorno por Uso de Sustancias (TUS) puede ser un factor de riesgo de contagio (Dunlop et al., 2020; Volkow, 2020). La solución para continuar proporcionando los servicios fue el uso de la tecnología móvil. La crisis sanitaria evidenció un procedimiento que desde hace décadas existía, pero al cual no se le había prestado suficiente atención: la telemedicina o telesalud.

\section{ANTECEDENTES}

El concepto de telemedicina data de 1905 cuando un médico holandés utilizó el teléfono para transmitir y monitorear sonidos cardiacos. En 1959 la Universidad de Nebraska empleó la televisión interactiva para transmitir exámenes neurológicos, lo que se considera la primera consulta por video en tiempo real. A esto siguió la conformación de la Telepsiquiatría, a través de consejería a distancia.

La Organización Mundial de la Salud (s.f.) ha identificado la telesalud mental como un elemento clave para proporcionar atención; la cual utiliza dispositivos como el teléfono, correo electrónico, chat, mensajes de texto o videconferencia. Cuando el intercambio de información se da en tiempo real se denomina sincrónica, como ocurre en la videoconferencia o llamada telefónica; cuando la comunicación sucede en un espacio y tiempo distinto se designa como asincrónica y se lleva a cabo a través de correos electrónicos, WhatsApp y mensajes de texto (Torous et al., 2020).

El ciberespacio permite un encuentro social en tiempo real o diferido, crea un espacio temporal flexible donde la interacción puede acercarse o distanciarse según se desee. En las comunicaciones sincrónicas, paciente y terapeuta están al mismo tiempo en el teléfono o en la computadora, lo que facilita una comunicación directa, creando una sensación de presencia e intimidad en el surgimiento de ideas y sentimientos.

Un elemento común a las relaciones de texto sincrónicos y asincrónicos es la desinhibición, ya que las personas pueden expresarse más abiertamente debido al anonimato. Otra ventaja es que la distancia geográfica no es una barrera para acceder al otro.

Pero, ¿es posible proporcionar una intervención psicológica y psicoterapia a distancia? Los objetivos son menos delimitados que en una intervención breve; a través de ellas se buscan cambios en el estado mental, emociones y/o conducta. Múltiples investigaciones llegan a la conclusión que el factor fundamental para alcanzar la mejoría o curación es la calidad de la relación terapéutica, además de los elementos metodológicos y técnicos apropiadamente utilizados (Flückige et al., 2018; Kallergis, 2019). En la modalidad de terapia a distancia cabe preguntarse ¿cuál es el vínculo relacional que se establece con una imagen, una voz o un texto? ¿Como impacta el no mirar al otro a la construcción de la alianza terapéutica? Diversos estudios concluyen que no hay diferencia entre la relación que se establece a distancia por videoconferencia que cara a cara (Rees $\&$ Stone, 2005) aunque sigue sin contestarse esta pregunta en las relaciones de texto.

Desde que el servicio de Internet se estableció alrededor de 1990 la atención psicológica empezó a aparecer por este medio, aunque la mayoría de las personas se mostraban cautas con esta nueva forma de intervención. No obstante, en la medida que el Internet se expandía y el servicio de telesalud mental se ampliaba, iniciaron múltiples estudios para conocer su aceptación y eficacia. La aplicación de la terapia a distancia ha mostrado su eficacia en la prevención del suicidio, ansiedad, depresión, Trastorno por Déficit de Atención e Hiperactividad (TDAH), trastornos de conducta y desorden de estrés postraumático, entre otros (Aboujaqude et al., 2015; Chakrabarti, 2015; Heravian \& Chang, 2018).

\footnotetext{
Autor de correspondencia:

Ricardo Sánchez Huesca, Director Normativo. Centros de Integración Juvenil.

Correo electrónico: direccionnormativa@cij.gob.mx y sanchezhuesca_ricardo@hotmail.com

doi: 10.28931/riiad.2021.1.07
} 


\section{TERAPIA A DISTANCIA CON USUARIOS DE SUSTANCIAS}

De acuerdo con el Informe Mundial sobre las Drogas 2020, de la Oficina de las Naciones Unidas contra la Droga y el Delito, en el mundo una amplia cantidad de personas con TUS requiere tratamiento, pero sólo una cantidad reducida lo recibe. La telesalud mental es un recurso que podría ayudar a resolver esta necesidad.

Incluso cuando la implementación de intervenciones para usuarios de drogas a través de Internet comenzó a desarrollarse desde los años 2000, la atención en salud mental a distancia se había estado aplicando desde mucho tiempo antes. En México, la atención a distancia con usuarios de sustancias inició en 1978 con el Servicio de Orientación e Información Telefónica de los Centros de Integración Juvenil (CIJ), institución que al observar que personas usuarias y sus familiares no podían acceder a recibir atención cara a cara, crean este servicio telefónico. Desde entonces y hasta la actualidad, se continúa ofreciendo orientación y soporte psicológico por teléfono, ahora ampliado con mensajería instantánea (Centros de Integración Juvenil, 2019). A causa de la pandemia, los 110 centros de consulta externa especializada y las 9 unidades de hospitalización de los $\mathrm{ClJ}$ reestructuraron su servicio de consulta para proporcionarlo a distancia, por teléfono y en la web. El Instituto Nacional de Psiquiatría también lleva a cabo una intervención cognitiva-conductual a distancia para usuarios de drogas (Tiburcio et al., 2018).

A continuación, se describen algunos métodos de tratamiento a distancia específicos por sustancia:

\section{Alcohol}

El tratamiento para esta sustancia es el que ha recibido mayor atención. Un meta-análisis de los resultados de 19 ensayos clínicos (14,198 participantes) encontró que las intervenciones a través de internet para adultos son eficaces para disminuir el consumo semanal de copas estándar y respetar los límites de consumo recomendados. Las intervenciones que incorporaron la interacción con un profesional mostraron un impacto mayor que las que sólo fueron automatizadas (Riper et al., 2018).

Deady y colaboradores (2016) condujeron un ensayo clínico aleatorizado con 104 jóvenes de entre 18 y 25 años de edad con síntomas leves de depresión y consumo de alcohol en riesgo. El programa es una intervención cognitivo-conductual automatizada. Al término, los participantes que recibieron la intervención presentaron mejorías en la severidad de los síntomas de depresión y reducciones en la cantidad y frecuencia del consumo en comparación con el grupo control. Estas diferencias no fueron estadísticamente significativas en el seguimiento a seis meses.

\section{Tabaco}

Taylor y colaboradores (2017) realizaron un meta-análisis para determinar la efectividad de los tratamientos online para dejar de fumar. Incluyeron 67 ensayos clínicos aleatorizados (45,194 participantes). Los programas interactivos (con la guía de un terapeuta) y personalizados fueron más eficaces en promover la cesación del consumo.

En México, la utilización del tratamiento por Internet ha sido poco explorada. Un estudio evaluó una herramienta computarizada para promover el cese del consumo. El programa recababa información sobre el participante y lo guiaba a través de decisiones sobre apoyo farmacológico y consejería. Participaron 164 adultos. En el seguimiento a tres meses dos personas de cada 10 dejaron de fumar (Cupertino et al., 2018).

\section{Cannabis}

En Australia, Gates y colaboradores (2012) crearon un programa telefónico de cuatro sesiones semanales conducidas por consejeros; incorpora elementos de Entrevista Motivacional (EM) y cognitivo-conductuales a fin de que el consumo se reduzca gradualmente. Esta intervención ayuda a reducir la dependencia a cannabis y promueve la abstinencia en un corto plazo.

Otro programa autogestivo y llevado a cabo por Internet se compone de seis módulos que incluyen retroalimentación; reestructuración cognitiva y entrenamiento en habilidades, y prevención de recaídas. El sitio web hace un fólder personalizado para cada participante y automáticamente genera correos electrónicos para motivarlo. Los autores concluyen que esta intervención puede ser un medio eficaz para usuarios de cannabis no complicados (Rooke et al., 2013).

En Brasil, Fernandes y colaboradores (2010) evaIuaron la eficacia de la Intervención Breve Motivacional por teléfono para disminuir el consumo. El estudio incluyó 524 participantes distribuidos en dos grupos, a uno se le proporcionó la intervención completa y al otro una intervención mínima. A los seis meses, los participantes del grupo experimental mostraron mayor nivel de abstinencia.

Un estudio en Alemania sobre el efecto de las intervenciones en chat sincrónicas y asincrónicas de un programa para reducir el consumo, encontró que el mensaje sincrónico construye una alianza terapéutica más fuerte y una mayor satisfacción con el tratamiento (Jonas et al., 2018). 


\section{Cocaína}

Un ensayo clínico evaluó una intervención cognitivo-conductual automatizada, anónima, basada en EM y prevención de recaídas y la comparó con la intervención del grupo control, que consistió de módulos de información en línea. Se encontró que no hubo diferencias en el nivel de consumo entre los participantes que recibieron la intervención automatizada y los del grupo control, sin embargo, los primeros mostraron mayores niveles de retención (Schaub et al., 2019).

Carroll y colaboradores (2014) llevaron a cabo un ensayo clínico aleatorizado con usuarios dependientes de cocaína para evaluar la eficacia de una intervención cognitivo-conductual computarizada en comparación con el mantenimiento sólo con metadona. Los participantes asignados a la intervención computarizada fueron más propensos a lograr tres o más semanas de abstinencia y este efecto se mantuvo a los seis meses de seguimiento. En un análisis secundario, se exploró la eficacia de esta intervención para desarrollar estrategias de afrontamiento, encontrando que mejora dichas estrategias en situaciones de riesgo de consumo (Kiluk et al., 2017).

Freedman y colaboradores (2006) condujeron una investigación para evaluar el consumo de cocaína/crack, su craving y sus disparadores en personas en situación de calle a través de un sistema de llamadas automatizadas a un celular que se les proporcionó. La información se contrastó con pruebas de toxicología en orina, el resultado mostró $73 \%$ de coincidencia entre ellas, lo que sugiere que el uso de esta herramienta es adecuado para evaluar tanto el consumo como los disparadores del mismo. Además, está en evaluación el uso de un reloj "inteligente" que mediante un sensor permite la detección del consumo de cocaína, el cual es un método menos invasivo que las muestras de orina (Holtyn et al., 2019).

\section{Heroína y Opioides}

En un estudio con pacientes atendidos con metadona, Eibl y colaboradores (2017) analizaron el nivel de retención de 3,733 pacientes que fueron atendidos a través de telesalud, cara a cara o en una combinación de ambas. Aquellos que habían recibido el tratamiento predominantemente a través de telesalud, presentaron mayores niveles de retención a un año de haber iniciado el tratamiento.

Otra investigación exploró el uso del internet para la prescripción de buprenorfina en personas que viven en zonas rurales. Este medio es viable para los consumidores de opiáceos con dificultad para acudir presencialmente a una clínica (Brunet et al., 2020). En un estudio para evaluar los resultados del uso de videoconferencia para la prescripción de buprenorfina, Zheng y colaboradores (2017) hallaron que no existen diferencias entre el uso de esta modalidad y la intervención cara a cara en los días de abstinencia.

\section{Metanfetaminas}

Ameri y colaboradores (2019) realizaron una revisión sistemática sobre la utilidad de mensajes de texto y de aplicaciones de teléfono inteligente para disminuir el uso de metanfetaminas y conductas sexuales de riesgo en hombres que tienen sexo con hombres. Encontraron que éstos son eficaces para reducir el consumo y las relaciones sexuales sin protección.

Otro estudio evaluó el contenido de las publicaciones en redes sociales de grupos dedicados a apoyar la recuperación de personas con consumo. Las publicaciones ofrecían información sobre la sustancia, mensajes de contención emocional y formación de redes de apoyo. Sugieren que este tipo de mensajes puede ser útil para disminuir el estigma que conlleva el uso de la droga y conectar con otras personas que puedan servir como modelos de apoyo (Ellway et al., 2019).

También se ha abordado la utilidad de una aplicación para celular que busca promover la mejora en déficits cognitivos, como resultado del consumo. Completar las tareas de la aplicación se asocia con una mejoría en el control de impulsos y en las habilidades intelectuales de los participantes (Zhu et al., 2018).

\section{Implicaciones técnicas, éticas y de privacidad}

En 2018, en América Latina y el Caribe, 13 de cada 100 personas contaban con una suscripción a banda ancha (Banco Mundial, 2018). En México, hay 80.6 millones de usuarios de Internet y 86.5 millones de usuarios de celular, sólo $44.3 \%$ de los hogares dispone de una computadora. Los usuarios de Internet de la zona urbana ascienden a $76.6 \%$, mientras que en la rural llegan a $47.7 \%$ (Instituto Nacional de Estadística y Geografía, 2020). Estas disparidades en el acceso a herramientas tecnológicas en México y en países en vías de desarrollo es un obstáculo a resolver para garantizar el acceso a tratamientos a distancia (Galea-Singer et al., 2020; Pereira-Sánchez et al., 2020).

Por otra parte, el intercambio de datos sensibles como hábitos, patrón de consumo y trastornos concomitantes exige la protección y manejo adecuado para evitar su divulgación o uso perjudicial (Chaet et al., 2017). Es necesaria la creación de políticas de protección de datos que incluya el desarrollo de tecnologías que aseguren que la transmisión de información es segura. Cuando la persona 
atendida es un menor de edad, los padres o tutores deben dar su consentimiento informado.

También es necesario asegurar la privacidad del espacio en donde se proporciona la consulta a distancia. ¿Cuál es el concepto de privacidad en una sesión terapéutica virtual, llevada a cabo en casa, donde probablemente estén presentes otros miembros del grupo familiar? ¿Cuáles son los temas íntimos posibles de exponer a través de medios virtuales? ¿Cómo podría trabajarse la violencia doméstica desde el propio espacio donde ocurre? Habrá que resolver éstas y otras preguntas en esta apertura al trabajo a distancia.

\section{CONCLUSIONES}

La pandemia por COVID-19 alteró completamente la forma de vida, se han tenido que descubrir formas diferentes de relación. La incorporación de las nuevas tecnologías a la salud mental presenta innumerables posibilidades: abre la oportunidad de atender a poblaciones difíciles de alcanzar por motivos geográficos o económicos.

Una de las experiencias enriquecedoras que dejará esta pandemia es el posicionamiento de los recursos digitales, que seguramente se incorporarán a los programas de salud mental de manera exclusiva o híbrida de acuerdo con los recursos y necesidades específicas del paciente, terapeuta y localidad geográfica. Ante ello, habrá que considerar la formación académica necesaria para proporcionar tratamientos en línea con la rigurosidad de los entrenamientos presenciales en salud mental y adicciones, los cuales además del cumplimiento de una currrícula teórica, incluyen supervisión de casos por otros especialistas.

Pese a que haya mostrado eficacia en muchas de sus evaluaciones, el tratamiento a distancia con personas consumidoras de sustancias requiere de mayor evidencia debido a la diversidad y amplitud de poblaciones que impacta. Es necesario que el análisis sea a través de ensayos clínicos aleatorizados, con muestras amplias y representativas, con usuarios de diferentes sustancias y condiciones como sexo, edad, escolaridad y estatus socioeconómico.

La telesalud mental en adicciones y otros trastornos mentales es un campo clínico y de investigación con múltiples interrogantes y con amplias oportunidades.

\section{REFERENCIAS}

Aboujaqude E., Salame W., Naim L. (2015).Telemental halth: a status update. World Psychiatry, 14(2), 223-230. doi: 10.1002/ wps.20218

Ameri, A., Keshvardoost, S. \& Bahaadinbeigy, K. (2019). Impact of Mobile Phone-Based Interventions on Methamphetamine Use and High-risk Sexual Behaviors in Men Who Have Sex with Men (MSM): A Systematic Review. Addiction and Health, 12(1), 58-68.

Banco Mundial. (2018). Recuperado de https://datos.bancomundial. org/indicator/IT.NET.BBND.P2.

Brunet. N., Moore, D. T., Lendavai Wischik, D., Mattocks, K.M. \& Rosen, M. I. (2020). Increasing buprenorphine access for veterans with opioid use disorder in rural clinics using telemedicine. Substance Abuse, 1-8. doi: 10.1080/08897077.2020.1728466

Carroll. K. M., Kiluk, B. D., Nich, C., Gordon, M. A., Portnoy, G. A., Marino, D. R. \& Ball, S. A. (2014). Computer-Assisted Delivery of Cognitive-Behavioral Therapy: Efficacy and Durability of CBT4CBT Among Cocaine-Dependent Individuals Maintained on Methadone. American Journal of Psychiatry, 171, 436-444. doi: 10.1176/appi.ajp.2013.13070987.

Centros de Integración Juvenil, A. C. (2019). 50 años de atención a las adicciones en México: La experiencia de Centros de Integración Juvenil. CIJ.

Chaet, D., Clearfield, R., Sabin, J. \& Skimming, K. (2017). Ethical practice in Telehealth and Telemedicine. Journal of General Internal Medicine, 32(10), 1136-1140. doi: 10.1007/s11606-017-4082-2.

Chakrabarti, S. (2015). Usefulness of telepchyatry: A critical evaluation of videoconferencing-bases aproches. World Journal of Psychyatry, 5(3), 286-304. doi: 10.5498/wjp.v5.i3.286.

Cupertino, A. P., Cartujano-Barrera, F., Perales, J., Formagini, T., Rodríguez-Bolaños, R., Ellerbeck, E. F., Ponciano-Rodríguez, G. \& Reynales-Shigematsu, L. M. (2018). "Vive Sin Tabaco. ¡Decídete!" Feasibility and Acceptability of an e-Health Smoking Cessation Informed Decision-Making Tool Integrated in Primary Healthcare in Mexico. Telemedicine and e-Health, 25(5), 425431. doi: 10.1089/tmj.2017.0299.

Deady, M., Mills, K.L., Teeson, M. \& Kay-Lambkin, F. (2016). An Online Intervention for Co-Occurring Depression and Problematic Alcohol Use in Young People: Primary Outcomes From a Randomized Controlled Trial. Journal of Medical Internet Research, 18(3), 1-12. doi: 10.2196/jmir.5178.

Dunlop, A., Lokuge, B., Masters, D., Sequeira, M., Saul, P., Dunop, G., Ryan, J., Hall, M., Ezrad, N., Haber, P., Lintzeris, N. \& Maher, L. (2020). Challenges in maintaining treatment services for people who use drugs during the COVID-19 pandemic. Harm Reduction Journal, 17(26). doi: 10.1186/s12954-020-00370-7

Eibl, J. K., Gauthier, G., Pellegrini, D., Daiter, J., Varenbut, M., Hogenbirk, J. C., \& Marsh, D. C. (2017). The effectiveness of telemedicine-delivered opioid agonist therapy in a supervised clinical setting. Drug and Alcohol Dependence, 176, 133-138. doi: 10.1016/j.drugalcdep.2017.01.048.

Ellway, D., Reilly, R., Le Couteur, A. \& Ward, J. (2019). Exploring How People Affected by Methamphetamine Exchange Social Support Through Online Interactions on Facebook: Content Analysis. JMIR Mental Health, 6(10), 1-12. doi: 10.2196/14011.

Fernandes, S., Ferigolo, M., Canellas, M., Moreira, T. C., Sngalli, P., Galvao, C. \& Tannhauser, H. (2010). Brief motivacional inerventon and telemedicine: A new perspective of treatment to marijuana users. $\mathrm{Ad}$ dictive Behaviors 35, 750-755. doi: 10.1016/j.addbeh.2010.03.001. 
Flückige, C., Del Re, A. C., Wampold, B. \& Horvat, A. O. (2018). The Alliance in Adult Psychotherapy: A Meta-Analytic Synthesis. Psychotherapy, 55(4), 316-340. doi: 10.1037/pst0000172

Freedman, M., Lester, K. M., McNamara, C., Milby, J. B. \& Schumacher, J. E. (2006). Cell phones for ecological momentary assessment with cocaine-addicted homeless patients in treatment. Journal of Substance Abuse Treatment 30,105-111. doi: 10.1016/j. jsat.2005.10.005

Galea-Singer, Newcombe, Farnsworth-Grodd, Sheridan, Adams \& Walker (2020). Challenges of virtual talking therapies for substance misuse in New Zealand during the COVID-19 pandemic: an opinion piece. New Zealand Medical Journal, 133(1515), 104-111.

Gates, P. J., Norberg, M. M., Copeland, J. \& Digiusto, E. (2012). Randomized controlled trial of a novel cannabis use intervention delivered by telephone. Addiction, 107(12), 2149-2158. doi: 10.1111/j.1360-0443.2012.03953.x

Heravian A. \& Chang B. (2018). Mental health and telemedicine in the acute care setting: Applications of telepsychiatry in the ED. The American Journak of Emmergency Medicine, 36(6), 118-119.

Holtyn, A. F., Bosworth, E., Marsch, L. A., McLeman, B., Meier, A., Saunders, E. C., Ertin, E, Ullah, S. A., Hossain, M., Kumar, S., Preston, K. L., Vahabzadeh, M., Shmueli-Blumberg, D., Collins, J., McCormack, J., \& Ghitza, U. E. (2019). Towards detecting cocaine use using smartwatches in the NIDA clinical trials network: Design, rationale, and methodology. Contemporary Clinical Trials Communications, 15, 100392. doi: 10.1016/j.conctc.2019.100392.

Instituto Nacional de Estadística y Geografía. (2020). Comunicado de prensa núm. 103/20. México: INEGI. Recuperado de https:// www.inegi.org.mx/contenidos/saladeprensa/boletines/2020/OtrTemEcon/ENDUTIH_2019.pdf.

Jonas, B., Tensil, M. D., \& Tossman, P. (2018). Effects of treatment length and chat-bases counseling in a Web-bases intervention for cannabis users: randomized factorial trial. Journal of medical internet research, 20(5), e166. doi: 10.2196/jmir.9579.

Kallergis, G. (2019). The contribution of the relationship between therapist-patient and the context of the professional relationship. Psychiatriki, 30(2), 165-174.

Kiluk, B. D., DeVito, E. E., Buck, M. M., Hunkele, K., Nich, C. \& Carroll, K. M. (2017). Effect of computerized cognitive behavioral therapy on acquisition of coping skills among cocaine-dependent individuals enrolled in methadone maintenance. Journal of Substance Abuse Treatment, 82, 87-92. doi: 10.1016/j. jsat.2017.09.011.

Oficina de las Naciones Unidas Contra la Droga y el Delito. (2020). World Drug Report. Resumen Ejecutivo. World Drug Report 2020. United Nations.

Organización Mundial de la Salud. (s.f.). E-health technologies and substance abuse. Recuperado de http://www.who.int/substance_abuse/activities/ehealth/en

Pereira-Sánchez, V., Adiukwu, F., El Hayek, S., Gashi Bytyçi, D., Gonzalez-Diaz, J.M., Kudva Kundadak, G., Larnaout, A. \&
Nofal, M. (2020). COVID-19 effect on mental health: patients and workforce. The Lancet, 7(6), E29-E30. doi: 10.1016/S22150366(20)30153-X

Rees C., \& Stone, S. (2005). Therapeutic Alliance in fase-to-fase versus videoconference psychotherapy. Professional Psychology Research and Practice, 36, 649-653.

Riper, H., Hoogendoorn, A., Cuijpers, P., Karyotak,i E., Boumparis, N., Mira, A., Andersson, G., Berman, A. H., Bertholet, N., Bischof, G., Blankers, M., Boon, B., Boß, L., Brendryen,, H., Cunningham, J., Ebert, D., Hansen, A., Hester, R., Khadjesari, Z., Kramer, J. ... Smit, J. H. (2018). Effectiveness and treatment moderators of internet interventions for adult problem drinking: An individual patient data meta-analysis of 19 randomised controlled trials. PLoS Med 15(12), e1002714. doi: 10.1371/journal. pmed. 1002714

Rooke, S. E., Copeland, J., Norberg, M. N., Hine, D. W. \& McCambridge, J. (2013). Effectiveness of a Self-Guided Web-Based Cannabis Treatment Program: Randomized Controlled Trial. Journal of Medical Internet Research 15(2), e26. doi: 10.2196/jmir.2256.

Schaub, M., Sullivan, R., Haug, S. \& Stark, L. (2019). Web-Based Cognitive Behavioral Self-Help Intervention to Reduce Cocaine Consumption in Problematic Cocaine Users: Randomized Controlled Trial. Journal of Medical Internet Research, 14(6), e166. doi: 10.2196/jmir.2244

Taylor, G.M.J., Dalili, M. N., Semwal, M., Civljak, M., Sheikh, A. \& Car, J. (2017). Internet-based interventions for smoking cessation. Cochrane Database of Systematic Reviews, 9, 1-156. doi: 10.1002/14651858.CD007078.pub5.

Tiburcio, M., Lara, A., Martínez, N., Fernández, M., \& Aguilar, A. (2018). Web-Based intervention to reduce substance abuse and depresión: a Tree arm randomized trial in Mexico. Substance use \& misuse, 53(13), 2220-2231. doi:10.1080/1082608 4.2018.1467452.

Torous, J., Myrick, K. J., Rauseo-Ricupero, N., \& Firth, J. (2020). Digital Mental Health and COVID-19: Using Technology Today to Accelerate the Curve on Access and Quality Tomorrow. JMIR mental Health, 7(3), 1-6.

Volkow, N. (2020). Collision of the COVID-19 and Addiction Epidemics. Annals of Internal Medicine. doi:10.7326/M20-1212.

Zheng, W., Nickasch, M., Lander, L., Wen, S., Xlao, M., Marshalek, P., Dlx, E. \& Sullivan, K. (2017). Treatment Outcome Comparison between Telepsychiatry and Face-to-face Buprenorphine Medication-Assisted Treatment (MAT) for Opioid Use Disorder: A 2-Year Retrospective Data Analysis. Journal of Addiction Medicine, 11(2), 138-144. doi: 10.1097/ADM.0000000000000287.

Zhu, Y., Jiang., Su, H., Zhong, N., Li, R., Li, X, Chen, T., Tan, H., Du, J., Xu, D., Yan, H., Xu, D., \& Zhao, M. (2018). A Newly Designed Mobile-Based Computerized Cognitive Addiction Therapy App for the Improvement of Cognition Impairments and Risk Decision Making in Methamphetamine Use Disorder: Randomized Controlled Trial. JMIR mHealth uHealth, 6(6), 1-14. doi: 10.2196/10292. 